\title{
The Cleanliness of Parts and Components of Fluid Systems in Automotive, Hydraulics and Aerospace complying with ISO16232, ISO 18413 and ISO 12345
}

\author{
홍정희 · 마이크 데이 \\ Jeong-Hee Hong and Mike Day
}

\begin{abstract}
In order to improve the life and reliability of fluid components used in the Automotive, Hydraulics and Aerospace industries, the cleanliness of the system parts and components before their assembly into the system is being addressed. The measurement of components and parts cleanliness is related to contaminant collection, analysis and data reporting and ISO Standards are continually being developed to control procedures. This Paper explains the processes in these standards and explains how similar procedures have been adapted to suit the requirements of the above Industries. It also gives guidance and recommendations on the implementation of these standards, so that the best use can be made of them.
\end{abstract}

\section{Introduction}

This document is to summarize standard practices related to contaminant collection, analysis and data reporting for cleanliness evaluation of manufactured parts and components, specifically being applied to the fluid systems (power train, lube, fuel and hydraulics) in Automotive, in Fluid Power and in Aerospace markets.

\section{Contaminant Extraction Methods}

\subsection{Validating the extraction process}

Whatever method of extraction is used it is essential that the extraction process is validated. Both ISO12345 and ISO16232 define the extraction process to be validated when the extracted contamination, within six repeated extraction trials from the same item (part or component), is equal to or less than $10 \%$ of the cumulative contamination extracted. This is termed "the End Point" If not, the extraction is not working effectively and has to be changed and revalidated. It should be noted that this is not a feature of ISO18413. All extractions perform should be validated.

ISO18413 Table2 gives us advice on the most appropriate extraction method for a range of components

\subsection{Agitation Extraction Method}

The contaminants are extracted by partially filling the component with a known volume of test liquid, sealing its openings, and agitating or "sloshing" it in order to remove the particles from the controlled surfaces and suspend them in the test liquid for subsequent analysis. It is best suited for hollow components like hoses or pipes.

The efficacy of the agitation method depends on type of agitation, duration of agitation, choice of test liquid.

Applicable standards: ISO16232 part 2; ISO18413 Clause 5.3

홍정희: 한국폴(주) 응용기술연구소 이사

E-mail: Jeong-hee_Hong@Pall.com,Tel: 02-560-8791

ISO/TC131/SC6 \& ISO/TC20/SC10 KATS

Mike Day: CMS Consultants Ltd, UK

E-mail: mikejday31@gmail.com

ISO/TC131/SC6 \& ISO/TC20/SC10 BSI 


\subsection{Pressure rinse extraction method}

The contaminants are extracted by pressure rinsing from the controlled surfaces of the component with a jet of filtered test liquid which removes the particles from the surfaces and carries them away for subsequent analysis.

The pressure rinse liquid dispenser is used as a device for providing clean test liquid at a suitable pressure and a flow rate capable of extracting the particles in an effective manner. The efficacy of pressure rinsing depends on pressure, flow rate, distance, angle, shape, size and diameter of the nozzle, rinsing time and liquid volume per area unit.

Applicable standards: ISO12345 Clause 5.5.3; ISO16232 part 3; ISO18413 Clause 5.4

\subsection{Ultrasonic Vibration Extraction Method}

The contaminant is removed by subjecting the item to ultrasonic vibration. The principal characteristics of the ultrasonic equipment (notably power, frequency, and dimensions of the bath) should be specified in the test report and the inspection document. In general, by applying transducers to floor or wall surfaces of the bath, it is possible to achieve a high degree of homogeneous sonic distribution.

Applicable standards: ISO12345 Clause 5.5.3; ISO16232 Part4; ISO18413 Clause 5.5

\subsection{Functional Test Extraction (End use simulation) Method}

The component is installed in a validated test bench which is supposed to simulate the component's functional operation. The circulation of the liquid under known conditions detaches the contaminants from the controlled surfaces and transfers them to the test liquid, for subsequent analysis. The flow of the test liquid is achieved either by pressure or by vacuum. In the case of an active component, depending on the function principle, the component is actuated either by an external device or by the traversing liquid. In reality this test is a flushing bench as component manufacturers will rarely circulate the contaminant during a function test as this will damage the component.
Applicable standards: ISO12345 Clause 5.5.3; ISO16232 Part 5; ISO18413 Clause 5.6

\section{Protocol for Contaminant Extraction}

\subsection{Package during transportation}

If the particles detached during transportation of the test components and particles from packaging have to be included in the cleanliness test they shall be collected using appropriate extraction methods. During handling and storage of test components it shall be safeguarded that no contaminants are deposited on or removed from controlled surfaces. To prevent loss of particles during transport it may be necessary to seal openings in the test components with either tape or a suitable plug.

Applicable standard: ISO16232 Part3; ISO18413Clause 5.3

\subsection{Blank test}

A blank test is performed to verify that the environment, operating conditions, equipment and products used in the extraction procedure do not contribute a significant amount of contamination to the component analyzed. To ensure process consistency, a blank test should be performed at regular intervals using identical test parameters.

- For the determination of system blank values, identical conditions as the one applied during testing of the component is applied but with the component omitted (see 3.3)

- If the blank level exceeds $10 \%$ of the cleanliness of the component presumed or measured), then either the process is too dirty and re-cleaning is necessary, or the contamination level of the component is to low and it is necessary to increase the number of test components analyzed in order to collect more contaminants and thus fulfill the $10 \%$ limit.

\subsection{Protocol for validation of contamination extraction process}

The contamination extraction procedure should be validated. 
- Determine the most suitable extraction method and the operating parameters.

- Perform three extractions. For each of three samples (This is only for ISO16232 and there are no such instruction in ISO18413), establish either the total mass of contaminants or total number of particles. For particle concentrations, this should include total numbers of particles larger than the smallest particle sizes specified in the inspection document.

- Divide the result of last sample by the sum of all the results obtained

- If the value obtained is less than $10 \%$ of this sum, the end point is reached and the extraction is complete. The cleanliness level of the component is the sum of the extractions.

- If the value obtained > $10 \%$, further extraction is necessary. If six extractions have been performed without reaching a value $10 \%$ then the extraction parameters are not suitable and will have to be modified.

\section{Analysis Methods}

\subsection{General}

A variety of standard, contaminant analysis methods and data reporting formats are used to produce the required part or component cleanliness data.

The major difference separating ISO16232 and other standards is the requirement to analyze all of the extraction fluid so that all particles including the lager particles, typically at much lower concentrations, are not overlooked. Because of this need, the microscopic procedures of ISO 16232 are completely automated.

Both ISO16232 and ISO18413 describe four basic contaminant analysis methods: gravimetric, particles size, chemical composition, particle size distribution and largest particles size. ISO12345 includes gravimetric, particle size distribution and largest particle size. It also includes a Field Contaminant Monitor (FCM) for monitoring the effectiveness of cleaning process.

\subsection{Gravimetric Analysis Method}

Determines the weight of contaminant extracted from the component and deposited on a membrane filter. The contaminant is generally separated from the test liquid by filtration through a membrane filter under controlled conditions and the mass of contaminant is determined by subtracting the initial weight of the membrane filter from the final weight. ISO16232-3 stipulates that all of the contaminants collected from controlled surface is analyzed.

Applicable standards: ISO12345 Clause 6.3; ISO16232 Part 7; ISO18413 Clause 6.3; ISO4405

\subsection{Particle Counting by Microscope}

Here the contaminant in the extraction liquid is filtered through a membrane filter and deposited on its surface. After drying the membrane filter, its surface is observed using a microscope (usually optical) and the particles are sized and counted on the basis of their longest dimension. In the case of ISO18413, IS4407 is specified and there is no restriction on the pore size of the membrane filter proved that it retains particles of the minimum states size, nor the volume filtered provided that the aliquot is representative.

ISO4407 uses manual or automatic (Image Analysis). Statistical counting can mean that critical and larger particles may be missed.

In case of ISO16232, this analysis can also be performed manually or, but the requirement to count all the extraction fluid (this can mean multiple membranes) means that manual full-surface counting is impractical, as it is a tiring task associated with errors. ISO16232 also gives the option of counting using the SEM.

A major difference is seen here with ISO16232 and ISO4407 as ISO4407 presents the data as cumulative (particles greater than a given size) particle counts, whereas ISO16232 presents data in size intervals as differential counts. Although the data is convertible, the resulting codes are not comparable.

Applicable standards: ISO $12345 \quad$ Clause6.4 (ISO4407); ISO16232 Part 8; ISO18413 Clause 6.4 ; ISO 4407 


\subsection{Particle Nature Analysis (Chemical composition analysis)}

The contaminant collected from controlled surfaces and deposited on a membrane filter is examined to determine its chemical composition by means of appropriate instrumentation. For the ISO18413, the technique is not specified and any technique can be used, such as a manual assessment using an optical microscope, scanning electron microscope (SEM) equipped with energy dispersion $\mathrm{X}$-Ray emission spectroscopic analysis (EDX) or an X-ray fluorescence spectrometer (XRF).

In ISO16232, the chemical elemental composition is determined by SEM/EDX analysis only. With ISO16232-8 the particle size distribution 4.3) and particle nature can be performed at the same time.

Applicable standard: ISO16232 Part 8; ISO18413 Clause 6.5

\subsection{Particle Counting using an Automatic Particle Counter (Particle size distribution)}

In case of ISO18413, after obtaining the sample so as to contain a representative portion of the contaminants, the number and size of particles is determined by automatic optical particle counter using light extinction sensors (ISO11500).

In case of ISO16232, the particle size distribution of particulate contaminants extracted from automotive components is determined using automatic light extinction particle counter instruments (APC). This standard only applies to the analysis of the whole extraction fluid volume.

The size range of particles that can be measured by this technique is limited to $>70 \mu \mathrm{m}$ (c), because of the method of calibration used (ISO 11171). However, the calibration can be extended to other sizes provided that it is agreed and included in the Inspection Document.

The major difference between ISO 16232 and ISO18413 is that in 16232 the extracted fluid has to be transferred to an analysis reservoir where all the extracted fluid is passed through the sensor and analyzed.
This technique is only applicable to measuring particles contained in clear and single phase liquids.

ISO 12345 also calls for a Fluid Cleanliness Monitor.

Applicable Standards: ISO12345 Claus 6.4 (ISO4407); ISO16232 part 9; ISO18413 Clause 6.6(ISO11500)

\subsection{Largest Particle Size}

Some organizations require "largest" particles sizes data. This is determined by microscopic evaluation of the longest particles or particles based upon the largest dimension.

\section{Data presentation and Reporting}

There are major differences in the way that data is reported in the various standards, thus

- ISO12345 reports cumulative counts at $>15,>100$ and >200um particle sizes

- ISO16232 reports interval counts (differential counts) and specifies a range of sizes inspection document

- ISO18413 report cumulative counts, ISO16232 reports interval counts(differential counts)

- ISO12345 reports $>15,>100$ and $>200 \mu m$ particle sizes only

- ISO12345 has the FIECC comprising codes at $>200 />100 />15 \mu \mathrm{m}$

- The Component Cleanliness Code (CCC) for ISO16232 is written as a sequence, enclosed in parentheses and separated by slashes, of alphanumerical pairs specifying all or several of size classes. The capital letters $\mathrm{A}$ or $\mathrm{V}$ printed before the parentheses indicates if the code refers to $1000 \mathrm{~cm}^{\wedge} 2$ of wetted surface or to $100 \mathrm{~cm}^{\wedge} 3$ of wetted volume of the component.

- A similar code is being developed for the Fluid Power Industry under ISO/DIS21017.

\section{Conclusion}

These standards are used for auditing the 
cleanliness of components subsequent to the washing/cleaning process before their assembly into a system. The cleanliness level of components shall be managed by the specification established by two parties who are the supplier and end-user.

The following are recommended to ensure efficacy of the cleanliness evaluation process.

- When implementing audit of components, the contaminant extraction method should be reviewed contaminant extraction methods to ensure their efficacy and compliance to ISO16232 and ISO18413.

- All equipment extracting and analyzing particles from components should implement blank tests to verify or to validate the cleanliness of the equipment itself.

- If the blank level exceeds $10 \%$, it is necessary to either investigate the cleanliness of various procedures or if it is acceptable increase the number of test components analyzed in order to collect more particles and thus fulfill the $10 \%$ limit.

- The pressure of test liquid should be adjusted to obtain a jet of sufficient power to remove and transport the particles from the controlled surface without degrading or dissolving the surface material of the component. The selection of the nozzle is equally important and may have to be changed to suit the location e.g.) a needle shaped jet is used for drillings and narrow passages and a fan shaped nozzle is used for flat surfaces.

- For particle size distribution method, ISO18413 standard allows for dilution to facilitate microscopic counting, with the dilution factor.

- Extraction of contaminant of relative clean components like fuel pipes should be measured by ultrasonic extraction and pressure rinse extraction method and to be particle counting analysis methods rather than gravimetric method.

- To maintain appropriate cleanliness level of component hydraulic components like pump or valves are generally more cleanable, it is recommended to measure and analyze the cleanliness via particle counting methods using

\section{ISO16232 and ISO18413}

- Since hydraulic components like pumps or valves are generally more cleanable, it is recommended to measure and analyze the cleanliness via particle counting methods using ISO16232 and ISO18413

- Since wet lubricant components transmission and engine are usually more particle generation, it is recommended be measured with particle counting methods and gravimetric method ISO16232 and ISO18413

- In case of industrial hydraulic component cleanliness, the cleanliness requirement should be consistent with the known and/or anticipated function or application of the part or component. Typical reference sources for consideration in determining the cleanliness requirement for a particular part or component include: historical data existing company, industry, national, and international standards; functional performance, reliability, and durability requirements of part or component; and cleanliness specifications for similar parts or components.

- For fluid power system, ISO12669 is currently being drafted to give more consistent and logical approach to determining the cleanliness level required for modern systems.

\section{Reference}

[1] ISO 16232, Road Vehicles - Cleanliness of components of fluid circuits

[2] ISO12345, 1998 Diesel Engines -Cleanliness assessment of diesel fuel injection equipment

[3] ISO 4407, Hydraulic Fluid Power - Fluid Contamination - Determination of particulate contamination by the counting method using an optical microscope

[4] ISO 4406, Hydraulic Fluid power - FluidsMethod for coding the level of contamination by solid particles

[5] ISO 11943, Hydraulic Fluid power - On-Line automatic particle-counting system for liquids Methods of calibration and validation

[6] ISO 11171: 1999, Hydraulic fluid power -- 
Calibration of automatic particle counters for liquids

[7] IS0 11500: 1997, Hydraulic fluid power Determination of particulate contamination by automatic counting using the light extinction principle

[8] ISO/NP 12669: Hydraulic fluid power - Method for determining the required cleanliness level (RCL) for a system

[9] ISO/CD 21017: Hydraulic fluid power Cleanliness of Component and part - Expression of particulate contaminant level

[9] ISO 21018-3: Hydraulic fluid power Monitoring the level of particulate contamination of the fluid - Part3: Use of the filter blockage technique

\section{[Authors Introduction]} Jeong-hee Hong(홍정희 理事)

E-Mail:Jeong-hee_Hong@pall.com Tel.:02-560-8791, 010-9070-0669 Born on December 10, 1968.

Precision Mechanical Engineering in Busan National University, Bachelor's degree, on Feb. 1991. Precision Mechanical Engineering in Busan National University, Master's degree, on Feb.1993.

Worked at Research \& Development Center in Doosan Infracore; 1993-2000. Now working for Scientific \&Laboratory Service in Pall Korea Limited; 2000 2012. Represents Korea, ISO/TC131/SC6 \& ISO/TC20/SC10 Technical Committee Member in KATS

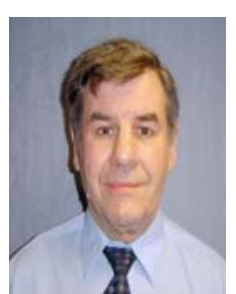

Mike Day

E-Mail: mikejday@gmail.com

Tel: +44 (0)2392 251658

Born on July 31, 1946.

Mechanical Engineering at Staffordshire University, Bachelor's degree on July 1970, Cranfield University, Master's degree on July 1984.

Worked for BHR Group Research 1974-1984, worked for Pall Europe Ltd., Scientific \& Laboratory Serviced, 1984- 2005 and Technology Group 2005-2011, Now managing director CMS Consultants Ltd.

Represents UK on ISO/TC131/SC6 and ISO/TC20/SC10. Convener of ISO/TC131/SC6/WG1. 